\title{
International Workshop on Prostatic Cancer and Benign Hypertrophy: Eine Konsensus-Konferenz
}

\author{
J. E. Altwein \\ Krankenhaus der Barmherzigen Brüder, München-Nymphenburg
}

Die amerikanische Krebsgesellschaft hatte eine viertägige Klausurtagung in einem Hotel mit dem bezeichnenden Namen "The Cloister” („Das Kloster“) ausgerichtet, um die vielschichtigen Probleme der Prostataerkrankungen zu behandeln und einen Konsens herzustellen. Zur benignen Prostatahypertrophie (BPH) wurden die nachstehenden Feststellungen getroffen, die sich in drei Themenkreise gliedern lassen:

\section{Problematik der BPH, Untersuchungsgang, Behandlungs- indikation und Beziehung der BPH zum Prostatakarzinom}

In der Konsensus-Kommission arbeiteten mit: W. Mebust, D. Bostwick (Pathologe an der Mayo Klinik), J. Grayhack, L. Holtgrewe, J. Wasson und R. Kirby (International Prostate Health Council).

Es ist unstrittig, daß die BPH inzwischen weltweit ein bedeutungsvolles Gesundheitsproblem darstellt, da die verursachte infravesikale Obstruktion zu einer funktionellen und anatomischen Detrusorschädigung, rezidivierenden Harnwegsinfekten, beeinträchtigter Nierenfunktion und letztlich zum Tode führen kann. Akute Auswirkungen sind der plötzliche Harnverhalt und rezidivierende Hämaturie. Obwohl $70 \%$ der über 70 jährigen eine histologisch nachweisbare BPH haben - die bereits mit 30 Jahren in der Prostata mikroskopisch nachgewiesen werden kann -, wirkt sich die BPH nur bei $30-40 \%$ obstruktiv auf die Blasenentleerung aus (Adenomkrankheit i.S. von C. E. Alken). Die Kosten der Therapie der Adenomkrankheit erreichen in den USA nahezu 4 Milliarden \$ pro Jahr (für die Bundesrepublik sind solche Berechnungen nicht möglich); die Umkehr der Alterspyramide wird zu einem weiteren Ansteigen der Behandlungskosten führen. Daher erschien der Konsensus der Kommission beachtenswert:

1. Eine sachgerechte Unterrichtung der Bevölkerung und Gesundheitsverantwortlichen zum BPH-Problem. Hierbei handelt es sich um eine Aufgabe der Gesundheitspolitik im weitesten Sinne.

2. Es müssen vermehrt wissenschaftliche Anstrengungen unternommen werden, um die Ätiologie, natürliche Entwicklung, Pathophysiologie und Maßnahmen zur Vorbeugung und Behandlung zu identifizieren. Dabei

Akt. Urol. 23 (1992) 131-135

(c) Georg Thieme Verlag Stuttgart · New York wurde Wert darauf gelegt, daß eine Patientenpräselektion für die verschiedenen Therapien erfolgt und auch Erkankungsprozesse, die eine Blasendysfunktion bewirken können, aufgezeigt werden. Die AUA trägt diesem Problem mit einer großen Verbundstudie Rechnung.

3. Kosten, Nutzen und Nachteil einer Behandlung der BPH und anderer Erkrankungen, die eine Blasendysfunktion bedingen können, müssen besonders sorgfältig ermittelt werden.

4. Es besteht ein hoher Bedarf an einem einheitlichen Berichtswesen über die Daten zur Behandlung der BPH. Neue Behandlungsmodalitäten sollten möglichst gegen ein Plazebo geprüft werden.

Der Kommission erschien es bemerkenswert, daß die transurethrale Prostataresektion (TURP) von Region zu Region mit unterschiedlicher Häufigkeit durchgeführt wird. In Erklärungen der Operationsindikation wurden Minimal-Forderungen an den untersuchenden Urologen formuliert: Neben einer sorgfältigen Anamnese sind eine aufzunotierende rektale Prostatapalpation, eine Harnuntersuchung und eine Bestimmung der Nierenfunktion (der Kreatininwert reicht aus) notwendig. Es wurde erheblich darüber diskutiert, ob routinemäßig bei Patienten mit einer signifikanten BPH das prostataspezifische Antigen (PSA) zu bestimmen sei. Man hat sich auf die vage Formulierung geeinigt, daß dies nur dann erforderlich sei, wenn zusätzliche Risikofaktoren für ein Prostatakarzinom bestehen. An welche man hierbei gedacht hatte, blieb offen.

Eine Anzahl von Patienten hat schließlich eine absolute Indikation zur Intervention, beispielsweise bei einem wiederholten Harnverhalt, rezidivierenden Harnwegsinfekten, einer wiederholten schweren Hämaturie oder bei einer BPH-bedingten Schädigung der Nieren oder der Harnblase. Demgegenüber wird aber eine größere Anzahl von Patienten wegen Miktionsbeschwerden medizinische Behandlung suchen. Bei der Bewertung dieser Symptome sollte der Schweregrad festgehalten, die Auswirkungen auf die Lebensqualität des Mannes ermittelt werden. Eine Anzahl von Fragebogen und Instrumenten zum Messen der Lebensqualität wurden inzwischen entwickelt und validiert. Die Kommission empfiehlt den Symptomen-Score, den Barry für die American Urological Association (AUA) entworfen hat und bei dessen Validierung die Reproduzierbarkeit der Befunde, die Unterscheidungsmöglichkeiten zwischen BPH- und Non-BPH-Patienten als günstig bewertet wurde. Darüber hinaus wurde dieser Symptomen-Score kürzlich von der Weltgesund- 
heitsorganisation ebenfalls angenommen. Werden Studienergebnisse veröffentlicht, dann sollte der Bericht die Ergebnisse dieses standardisierten Symptomen-Scores berücksichtigen.

Patienten mit milden Symptomen werden üblicherweise lediglich beobachtet, allerdings sollte eine stumme obstruktive Uropathie ausgeschlossen sein. Patienten mit mäßigen bis schweren Symptomen sollen so umfangreich untersucht werden, daß die Diagnose „klinisch relevante BPH mit Behandlungserfordernis“" abgeleitet werden kann. Bei diesen Untersuchungen handelt es sich um den Uroflow, Restharn und Urethrozystoskopie. Eine Untersuchung des oberen Harntraktes ist routinemäBig nicht erforderlich, ist aber dann indiziert, wenn der Patient eine schwere Hämaturie, rezidivierende Harnwegsinfekte, Steine oder andere Besonderheiten aufweist. Urodynamische Studien sind lediglich fakultativ zu vertreten.

Wenn die Indikation zur Behandlung gesichert ist, dann sollte der Urologe dem Patienten die verfügbaren medizinischen und chirurgischen Alternativen erläutern, so daß der Patient aktiv bei der Auswahl der Verfahren mitwirkt. Überraschend war die ausgesprochene Empfehlung, daß die Richtlinien zur Patientenbetreuung mindestens alle drei Jahre zu überprüfen sind und der Entwicklung angepaßt werden müssen.

Die Beziehung von Prostatahyperplasie und Prostatakarzinom ist trotz mancher Gemeinsamkeiten nicht so eng, daß eine eindeutige Progression von der BPH zum manifesten Prostatakarzinom erfolgt. Es gibt zwar verschiedene prämaligne Läsionen der Prostata (atypische adenomatöse Hyperplasie oder AAH und prostatische interepitheliale Neoplasie oder PIN), ohne daß der Nachweis dieser histologischen Veränderungen einen eindeutigen Hinweis auf das Fortschreiten zum Karzinom enthielten.

\section{Nicht-operative Behandlung der BPH}

Diese Konsensus-Kommission (L. Denis, $H$. Lepor, J. Geller, J. McConnel, T. Hald, K. Griffiths und M. Leishman) hatte große Schwierigkeiten, den gemeinsamen Nenner zu finden. Man gelangte zu der Auffassung, daß die Ätiologie der BPH unbekannt ist und alle Behandlungsformen palliativen Charakter haben. Der Prostatismus wird durch die infravesikale, BPH-bedingte Obstruktion oder die zunehmende Reizbarkeit der benachbarten Gewebe hervorgerufen. Diese „Reizbarkeit“ wird durch die Erwartungsangst des Patienten verstärkt, der eine Veränderung seines Miktionsverhaltens beobachtet. Es erschien allen unstrittig, daß die BPH als Erkrankung heterogen ist und sich ohne Bezug zum Volumen der BPH oder ihrer Form manifestiert. Erschwerend kommt hinzu, daß eine vergleichbare konstruktive und irritative Symptomatik auch durch andere Erkrankungen hervorgerufen werden kann. Auch die Blase verliert mit zunehmendem Alter ihre Reservoirfunktion und Fertigkeit, den Urin willkürlich $\mathrm{zu}$ entleeren. Im allgemeinen ist die klinische $\mathrm{BPH}$ nicht unmittelbar bedrohlich für den oberen Harntrakt, noch für das Überleben des Patienten. Es gibt gute Gründe, eine Beobachtung ohne Intervention als Alternative zu akzeptieren, wenn die Symptome minimal sind und die Lebensqualität somit kaum beeinträchtigt wird. Dabei ist besonders zu berücksichtigen, daß von dieser Gruppe von Patienten der geringe Prozentsatz von Komplikationen der TURP als unakzeptabel angesehen wird (Mortalität unter $1 \%$, Morbidität $16 \%$, Inkontinenz $0,8 \%$, Impotenz $5-10 \%$ und Reoperationsrate zwischen 6 und $15 \%$ im Verlaufe der folgenden 8 Jahre bei einer symptomatischen Verbesserung zwischen 88 und $95 \%$ ). Jede Alternative zur operativen Behandlung der BPH muß in Betracht gezogen werden und in Beziehung zu den Nebenwirkungen für den Patienten eingesetzt werden. Bei der Wertung der Behandlungsalternativen ist es eine Frage des Common Sense, daß invasive Verfahren äquieffektiv wie die Prostatektomie und ihre geringe Komplikationsrate zu sein haben.

\section{Alternative Therapie der BPH}

Non-invasiv: Hormontherapie Alpha-Blocker

invasiv:

transrektale oder transurethrale
Hyperthermie
transurethrale Thermotherapie (TUMT)
Ballon-Dilatation
temporäre oder permanente Stents.

Werden die alternativen Behandlungsformen nach ihrem Wirkungsmechanismus unterschieden, dann würde man als dynamisch die Alpha-Blocker und die transrektale Hyperthermie bezeichnen, hingegen die Hormontherapie, Thermotherapie, Ballondilatation und Harnröhren-Stents als statisch.

Wenn die Therapieentscheidung getroffen wurde, dann ist die BPH diagnostisch gesichert, es besteht keine der absoluten Indikationen zur Operation, noch würde der Patient eine Operation nicht aushalten. Besonders betont wurde von der Kommission, daß der Patient am Anfang nach dem WHO-Symptomen- und Lebensqualitäts-Score ausgewertet wurde. Der Behandlungserfolg wird nicht nur an diesen Scores, sondern auch an dem Uroflow zusammen mit dem Flowvolumen als Minimalanforderung gemessen. Der Restharn hat nur nachrangige Bedeutung bei der Erfolgsbewertung. Besonders hervorgehoben wurde immer wieder, daß der Nutzen der Behandlung den Nachteil der Therapie überschreiten muß und daß das Kosten-Nutzen-Verhältnis ausgewogen sein sollte, dies gilt beispielsweise für die hohen Materialkosten mancher Stents oder die hohen Gerätekosten bei der Thermotherapie. Bevor eine Therapieempfehlung gegeben wird, sollten alle Behandlungsalternativen Phase I (Durchführbarkeit und Sicherheit), Phase II (Wirksamkeit und Indikationen) und Phase III (randomisierte Studien zum Nachweis der Behandlungseffizienz im Vergleich zu einem Kontrollarm) durchgeführt sein.

\section{Alleinige Überwachung}

Nachdem nur etwa jeder 4. der Männer mit einer symptomatischen BPH eine chirurgische Intervention benötigt, ist die alleinige Beobachtung des Patienten eine der möglichen Optionen. Es gibt über den natür- 
lichen Verlauf der alleinigen Überwachung nur spärliche Daten, beispielsweise beträgt die Wahrscheinlichkeit des akuten Harnverhaltes zwischen 5 und $50 \%$ - bezugnehmend auf 2 Studien. Man schätzt, daß etwa bei einem Drittel der Patienten eine symptomatische Besserung ohne Therapie erfolgt, so daß diese Modalität als aktive Behandlung dann einzustufen ist, wenn keine absolute Indikation zur Prostatektomie besteht. Im Rahmen der alleinigen Überwachung ist eine straffe Patientenführung notwendig, das Verhalten ist zu modifizieren (z.B. Einschränkung der Flüssigkeitszufuhr, Blasentraining etc.) unter Einsatz anticholinerg wirksamer Medikamente mit Patienten, die überwiegend an irritativen Miktionsbeschwerden leiden und zugleich geringe Restharnmengen haben, ist sinnvoll. Prostatotrope Phytotherapeutika werden in diesem Krankheitsstadium intensiv eingesetzt.

\section{$\alpha$-Rezeptor-Blocker}

Es gibt eine wissenschaftliche Grundlage für die Anwendung von Alpha-1-Rezeptor-Antagonisten, die sog. Alphablocker, für die Behandlung der BPH; denn das hyperplastische, obstruierende Gewebe der Prostata erhält in erheblichem Umfange glatte Muskelzellen, die ebenfalls in der Prostatakapsel und im Blasenhals auftreten, verantwortlich sind für die Obstruktion. Für die Spannung ist verkürzt formuliert der Alpha-Rezeptor mit verantwortlich. Darüber hinaus gibt es Hinweise, daß bei einer Blasenentleerungsstörung eine Zunahme der AlphaRezeptoren auftritt. Alphablocker bewirken die Entspannung der glatten Muskulatur, selbst wenn die Masse des obstruierenden Gewebes nicht vermindert wird, so ist die dynamische Komponente des Entleerungswiderstandes zu senken. Seit 1976 Caine erstmals die Alpha-Rezeptoren-Blocker einsetzte, wurden mindestens 25 Studien über die Wirkung der Alpha-Rezeptoren veröffentlicht. Die Mehrzahl dieser Untersuchungen kam zu der Schlußfolgerung, daß die Alpharezeptoren-Blocker sich überwiegend für die Kurzzeitbehandlung eignen. Kirby berichtete über eine wesentliche Verbesserung des Uroflows und des Symptomen-Scores von Patienten unter Prazosin, einem selektiven Alpha-1-Blocker, der zweimal täglich gegeben werden muß. Lepor hat die Ergebnisse von vier Studien mit dem Langzeit-Alphablocker, Terazosin, veröffentlicht. Von den 163 behandelten Patienten besserte sich der maximale Uroflow um $50 \%$, während der Symptomen-Score um $54 \%$ fiel. Der gleiche Autor hat in einer 313 Patienten enthaltenden Studie auf der AUA in Toronto (1991) berichtet, daß sich mit der 10-mg-Dosis Terazosin der maximale Uroflow um $56 \%$ gegenüber $25 \%$ in der PlazeboGruppe besserte. Eine $30 \%$ ige Abnahme der Symptome wurde bei $87 \%$ der Terazosin-Patienten erreicht im Gegensatz zu lediglich $38 \%$ der Plazebo-Exponierten. Schwindel und Müdigkeit traten bei etwa 10\% der Behandelten auf, orthostatische Hypotension war selten ein Problem, wenn die Dosis des Terazosins sorgfältig titriert, d.h. von 2 auf $10 \mathrm{mg}$ erhöht wurde.

Aber nicht alle Alphablocker-Studien hatten derartig optimistische Ergebnisse, in einer Phase-IIIStudie mit Alfuzosin wurde eine Verbesserung des Uroflows überhaupt nicht beobachtet, obwohl der Symptomen-Score statistisch signifikant absank. Es ist anzumerken, daß bisher noch nicht geklärt ist, ob die Erfolgser- gebnisse von längerer Dauer sind. Die Kommissionsmitglieder kommen zu dem Schluß, daß die Alphablocker-Behandlung der obstruktiven BPH eine akzeptable Maßnahme darstellt, wenn die Ergebnisse der veröffentlichten randomisierten Studien eindeutig belegen, daß der Nutzen größer ist als der Nachteil und das Medikament in dem jeweiligen Land zugelassen ist.

\section{Hormontherapie}

Patienten reagieren eindeutig auf einen Androgenentzug etwa in Form einer medikamentösen Kastration durch die Zufuhr von LHRH-Analoga, auch wenn die Abnahme des Prostatavolumens in nicht eindeutiger Beziehung zur Verbesserung der Symptome stand. Nachteil ist der Verlust der Libido und der Potenz. Über die Wirkung der Antiandrogene wie Cyproteronazetat und Flutamid gibt es einzelne anekdotische Hinweise über ihre symptomatische Wirkung bei der klinischen BPH. Dabei hat Cyproteronazetat den Nachteil, daß es als gestagen wirkendes Steroid LH und FSH unterdrückt und somit ebenfalls Libido und Potenz mindert. Andere Gestagene wie Megestrolazetat, Hydroxy-Progesteronazetat sowie Gestonoronkapronat haben eine ähnliche biologische Wirkung wie der Chlormadinonazetat-Abkömmling Cyproteronazetat und sind nur in preliminären Studien zur Behandlung der BPH eingesetzt worden.

Flutamid als nicht-steroidales oder reines Antiandrogen blockiert lediglich die Bindung von Testosteron und 5-Alpha-Dihydrotestosteron zu dem Androgenrezeptor in der Prostata. Die Potenz wird nicht beeinträchtigt. Langzeituntersuchungen fehlen.

Die Einführung des 5-Alpha-ReduktaseHemmers, Finasteride, wurde sehr optimistisch aufgenommen. Eine umfangreiche Phase-II-Studie mit Finasteride ergab, daß das Prostatavolumen abnimmt und einige Symptome der BPH abklingen. Der geringe, aber signifikante Anstieg des Testosteronspiegels hat eine schwache Wirkung auf Libido und Potenz, ein physiologischer Effekt ist eine Abnahme des Ejakulatvolumens.

\section{Stents}

Temporäre Stents (z. B. Nissenkorn-Spirale) sollen die Obstruktion durch die BPH überwinden. Diese temporären Stents sind indiziert bei Patienten, die eine Operation nicht aushalten würden, aber unter einem Harnverhalt leiden. Einzelne Autoren setzen die temporären Stents auch dann ein, wenn Patienten überwiegend irritative Miktionsbeschwerden haben, um zu sehen, wie der hyperreflektorische Detrusor sich bei Beseitigung der intravesikalen Obstruktion verhält. Besonders wichtig ist die Überwachung dieser Patienten, da die Stents wandern und verkalken können. Bei jedem zweiten Patienten ist ein ausreichendes bis zufriedenes klinisches Ergebnis zu erzielen.

Die permanenten Stents sind immer noch im Experimentalstadium und sollen die TURP ersetzen. Tatsächlich kann, dies zeigen vorläufige Ergebnisse, der permanente Stent die Blasenentleerungsstörung überwinden. Es fehlt eine Phase-III-Studie zwischen Stent und 
TURP. Besonderer Nachteil sind die hohen Kosten, das gilt besonders für den Wallstent.

\section{Hyperthermie}

Die rektale und transurethrale Hyperthermie bei Temperaturen bis zu $45^{\circ} \mathrm{C}$ bessert die Symptome bei zwei von drei Patienten, wohingegen die objektiven Parameter nicht gebessert werden. Diese Behandlungsform sollte auf Patienten mit irritativen Miktionsbeschwerden im Gegensatz zu obstruktiven beschränkt werden, geringen Restharn und ein Prostatagewicht unter 70 g. Es kommt nicht zu erkennbaren Gewebsveränderungen und der Wirkungsmechanismus ist unbekannt. Eine Langzeitüberwachung ist zu fordern. Es gibt eine kleine randomisierte Studie im Vergleich zum Plazebo, die einen Vorteil dieses Verfahrens zeigt (Zerbib; demgegenüber konnten Fabricius u. Mitarb. in einem größeren randomisierten Versuch diese Erfahrungen nicht bestätigen; Anmerkung des Referenten). In jedem Falle ist die Hyperthermie ungeeignet bei der Blasenhalsstenose, Vergrößerung des Mittellappens oder einer großen Prostata.

\section{Thermotherapie}

Wird die Temperatur über $45^{\circ} \mathrm{C}$ erhöht, kommt es zu objektiven Zeichen der Gewebsnekrose. Infolgedessen kommt es auch zu Harnverhalten bei $4-40 \%$ der Patienten. Die subjektiven Symptome bessern sich bei etwa zwei Dritteln und eine objektive Besserung wurde bei etwa einem Drittel beobachtet. Eine Studie (Strohmaier u. Mitarb. 1990) fand die Thermotherapie schlechter als die TURP. Phase-III-Studien sind dringend zu fordern. Es gelten die gleichen Kontraindikationen wie für die Hyperthermie.

\section{Ballondilatation}

Dieses Verfahren öffnet gewaltsam die prostatische Urethra (Divulsion). Es kann zu einem Einreißen der vorderen Kommissur kommen. Diese Methode kann bei einer kleinen Gruppe von Patienten nützlich sein, es ist aber kontraindiziert bei Patienten mit einem Mittellappen oder einer großen Prostata. Die Kurzzeit-Erfolgsergebnisse erreichen maximal $60 \%$. Reddy u. Mitarb. zeigten kürzlich, daß bei der Mehrzahl der Patienten nach zwei Jahren kein Erfolg mehr nachzuweisen war. Insgesamt hängt das Ergebnis erheblich von der Patientenpräselektion ab, die so eng ist, daß etwa $1 \%$ der Patienten mit einer symptomatischen BPH hierfür in Frage kommt $($ Dowd). Der Effekt ist kurz anhaltend bei etwa der Hälfte der Patienten und im Ergebnis unvorhersehbar. Es kommt nicht zur Inkontinenz, Blutung oder Impotenz. Die Ballons sind vergleichsweise teuer.

Zusammenfassend hat das Konsensus-Komitee festgestellt, daß keine der non-operativen Alternativen die Ergebnisse der TURP erreicht und daß die Sicherheit und Wirksamkeit der diskutierten Verfahren in Phase-III-Studien auch im Langzeitexperiment zu belegen ist.

\section{Operative Behandlung der BPH}

Dies wurde von Brendler, Schlegel, Dowd, Kirby und Zattoni diskutiert. Es ist überraschend, daß bei einem Verfahren wie der Adenomektomie, die transurethral oder offen durchgeführt werden kann und als Goldstandard zur Therapie der BPH angesehen wird, so viele Urologen sich für therapeutische Alternativen begeistern. Zweifelsohne gibt es Risikofaktoren der TURP wie ein Resektionsgewicht über $45 \mathrm{~g}$, eine Resektionszeit über 90 Minuten, ein akuter Harnverhalt, ein Patient über 80 Jahre oder ein Serumkeratininanstieg auf über $1,5 \mathrm{mg} / \mathrm{dl}$. Unabhängige Risikofaktoren, die in gleichem Sinne für die offene Prostatektomie zu gelten haben, sind nicht so sorgfältig untersucht.

Die Ergebnisse der operativen Behandlung der BPH wurden von der Agency for Health Care Policy für die Jahre 1960 bis 1990 in einer Übersicht aller relevanten Arbeiten überprüft.

Die Kommission folgerte auf der Basis dieser Daten (Tab. 1), daß die TUIP und TURP bei Prostatae unter $30 \mathrm{~g}$ äquieffektiv sei und stufte die TUIP als zu selten eingesetzt ein.

Tab. 1 Ergebnisse der operativen Behandlung der BPH bei Patienten mit mäßigem Prostatismus.

\begin{tabular}{|c|c|c|c|}
\hline & TUIP & TURP & $\begin{array}{l}\text { offene Prostat- } \\
\text { ektomie }\end{array}$ \\
\hline $\begin{array}{l}\text { Wahrscheinlichkeit einer } \\
\text { symptomatischen Besserung }\end{array}$ & $\begin{array}{l}80 \% \\
(78-83)\end{array}$ & $\begin{array}{l}88 \% \\
(75-96)\end{array}$ & $\begin{array}{l}98 \% \\
(94-99,8)\end{array}$ \\
\hline $\begin{array}{l}\text { Abnahme des Symptomen- } \\
\text { Scores in } \%\end{array}$ & $73 \%$ & $85 \%$ & $79 \%$ \\
\hline $\begin{array}{l}\text { maximaler Uroflow } \\
\text { vor Behandlung } \\
\text { nach Behandlung }\end{array}$ & $\begin{array}{r}8 \\
15\end{array}$ & $\begin{array}{r}8 \\
18\end{array}$ & $\begin{array}{r}8 \\
23\end{array}$ \\
\hline $\begin{array}{l}\text { Chance einer neuerlichen } \\
\text { Behandlungsbedürftigkeit } \\
\text { innerhalb von } 5 \text { Jahren }\end{array}$ & $\begin{array}{l}8,1 \% \\
(6,8-9,6)\end{array}$ & $\begin{array}{l}3,4 \% \\
(1,1-9,6)\end{array}$ & $\begin{array}{l}0,4 \% \\
(0,04-4,2)\end{array}$ \\
\hline
\end{tabular}

Insgesamt sind aber die Ergebnisse der drei operativen Verfahren deutlich besser als diejenigen der Behandlungsalternativen.

Die Komplikationsdichte betrug $14 \%$ nach der TUIP, 16,1\% nach der TURP und 21,7\% nach der offenen Prostatektomie. Bei den Komplikationen handelt es sich um Inkontinenz, Impotenz, retrograde Ejakulation, Schmerzen, erheblicher Blutverlust, Harnröhrenstriktur und Mortalität.

Die späten Komplikationen der transurethralen und offenen Prostatektomie wurden von Roos u. Mitarb. (1989) untersucht und im New England Journal of Medicine (320:1120-1124, 1989) veröffentlicht. Dies zeigte, daß sich die Spätmortalität nach TURP im Vergleich zur offenen Prostatektomie erhöhte mit einem relativen Mortalitätsrisiko 1,45 nach 8 Jahren. Die Daten, darüber waren sich viele Statistiker einig, können nur 


\begin{tabular}{llll}
\hline & Beobachtung & $\begin{array}{l}\text { Ballon- } \\
\text { Dilat. }\end{array}$ & $\begin{array}{l}\text { Alpha- } \\
\text { Blocker }\end{array}$ \\
\hline $\begin{array}{l}\text { Wahrscheinlichkeit einer } \\
\text { symptomatischen Besserung }\end{array}$ & $\begin{array}{l}42 \% \\
(31-55)\end{array}$ & $\begin{array}{l}57 \% \\
(37-76)\end{array}$ & $\begin{array}{l}74 \% \\
(59-86)\end{array}$ \\
$\begin{array}{l}\text { Abnahme des Symptomen- } \\
\text { Scores in \% }\end{array}$ & $<32 \%$ & $51 \%$ & $51 \%$ \\
$\begin{array}{l}\text { maximaler Uroflow } \\
\text { vor }\end{array}$ & 9 & 7 & 8 \\
$\begin{array}{l}\text { nach Behandlung } \\
\text { Chance einer neuerlichen }\end{array}$ & 8 & 13 & $\begin{array}{l}\text { (3) } \\
\text { Behandlungsbedürftigkeit } \\
\text { innerhalb von 5 Jahren }\end{array}$ \\
\end{tabular}

Tab. 2 Ergebnisse therapeutischer Alternativen zur Behandlung der BPH bei Männern mit mäßigem Prostatismus.

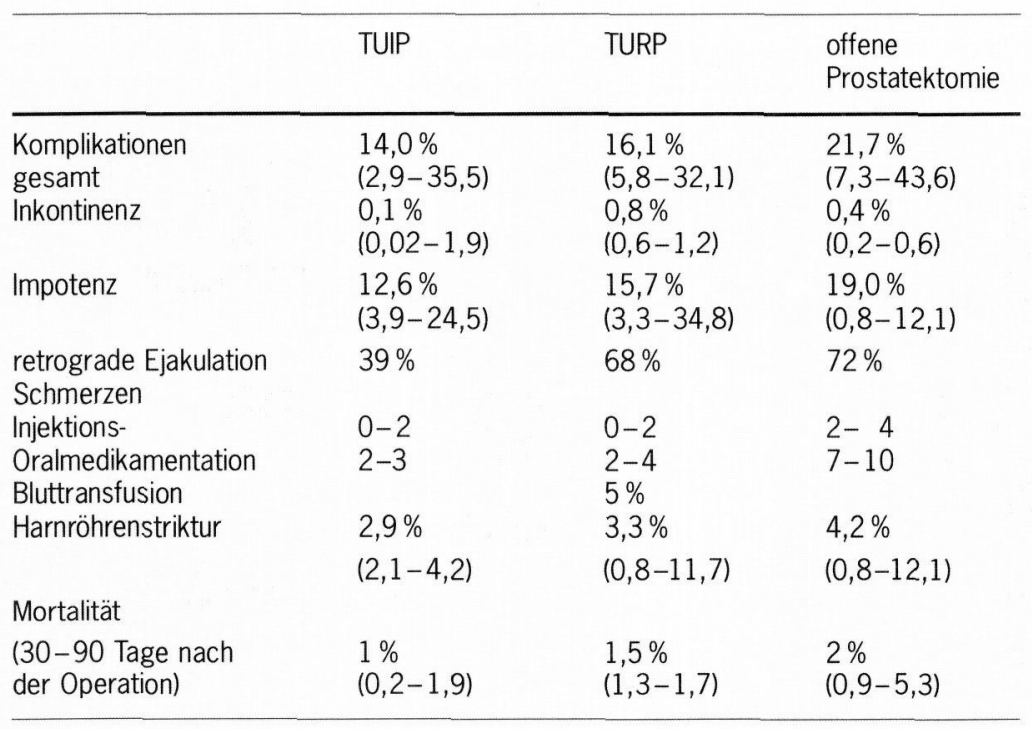

Tab. 3 Komplikationen der operativen Behandlung der $\mathrm{BPH}$. durch eine prospektive randomisierte Studie widerlegt werden (Tab. 3).

Zum Abschluß kam die Konsensus-Kommission auf die grundsätzlichen Probleme mit den therapeutischen Alternativen zu sprechen und faßte diese zusammen:

1. Es kommt nicht zu einer Untersuchung des Adenomgewebes, um ein Prostatakarzinom auszuschließen.

2. Der kurzzeitige Effekt der nicht-operativen Behandlung ist unvorhersagbar.

3. Die definitive Behandlung wird verzögert, die Kosten steigen, wenn eine Prostatektomie doch noch erforderlich werden sollte.

4. Hohe Gerätekosten, besonders bei der Hypothermie und Thermotherapie.

5. Der Langzeitnutzen und die Spätkomplikationen sind unbekannt.

Darüber hinaus müssen zusätzliche Probleme mit der medikamentösen Therapie der BPH Beachtung finden:

1. Die Medikamente mögen nicht leicht verfügbar in allen Regionen sein.
2. Unsichere Compliance der gerontologischen Patienten.

3. Verspäteter Beginn der pharmakologischen Wirkung (besonders bei der hormonalen Therapie).

4. Variable Medikamentenwirkung zwischen verschiedenen Patientengruppen.

5. Indikationen, Nebenwirkungen und medikamentöse Interaktionen sind $\mathrm{zu}$ beachten.

6. Hohe Kosten, wenn eine Langzeitbehandlung erforderlich ist.

Daraus ist insgesamt zu schließen, daß die Prostatektomie eine effektive definitive Behandlung der BPH darstellt. Urologen sollten sich ständig bemühen, die Komplikationsdichte auf das absolute Minimum zu senken und damit die Sicherheit der Patienten zu verbessern.

Prof.Dr. med. J. E. Altwein

Chefarzt d. Urologischen Abteilung

Krankenhaus der Barmherzigen Brüder

Romanstr. 93

8000 München 19 